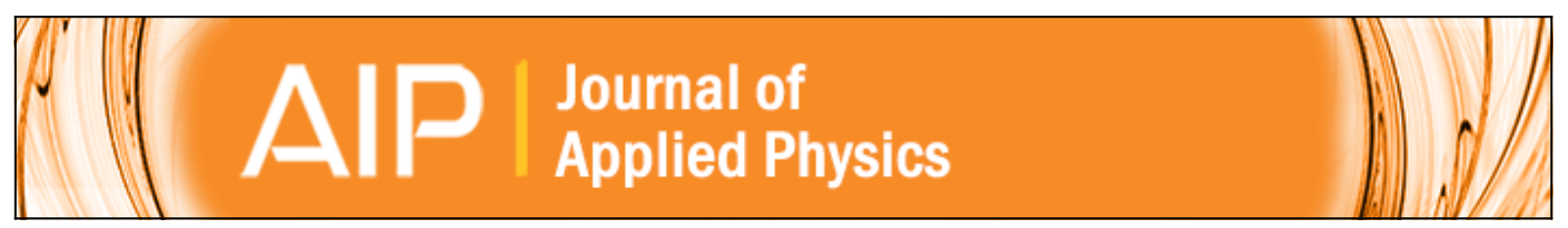

\title{
Magnetoelectric polymer nanocomposite for flexible electronics
}

M. Alnassar, A. Alfadhel, Yu. P. Ivanov, and J. Kosel

Citation: Journal of Applied Physics 117, 17D711 (2015); doi: 10.1063/1.4913943

View online: http://dx.doi.org/10.1063/1.4913943

View Table of Contents: http://scitation.aip.org/content/aip/journal/jap/117/17?ver=pdfcov

Published by the AIP Publishing

\section{Articles you may be interested in}

Multiferroic Ni0.6Zn0.4Fe2O4-BaTiO3 nanostructures: Magnetoelectric coupling, dielectric, and fluorescence J. Appl. Phys. 116, 124103 (2014); 10.1063/1.4896118

Magneto-electric effects in functionally stepped magnetic nanobilayers on ferroelectric substrates: Observation and theory on the influence of interlayer exchange coupling

J. Appl. Phys. 115, 193909 (2014); 10.1063/1.4878458

Converse magnetoelectric coupling in $\mathrm{NiFe} / \mathrm{Pb}(\mathrm{Mg} 1 / 3 \mathrm{Nb} 2 / 3) \mathrm{O} 3-\mathrm{PbTiO} 3$ nanocomposite thin films grown on $\mathrm{Si}$ substrates

Appl. Phys. Lett. 103, 192903 (2013); 10.1063/1.4828878

Observation of large magnetodielectric and direct magnetoelectric behavior in LCMO/PVDF 0-3 nanocomposites Appl. Phys. Lett. 102, 072902 (2013); 10.1063/1.4793307

Highly flexible poly (vinyldine fluoride)/bismuth iron oxide multiferroic polymer nanocomposites AIP Conf. Proc. 1447, 1309 (2012); 10.1063/1.4710494

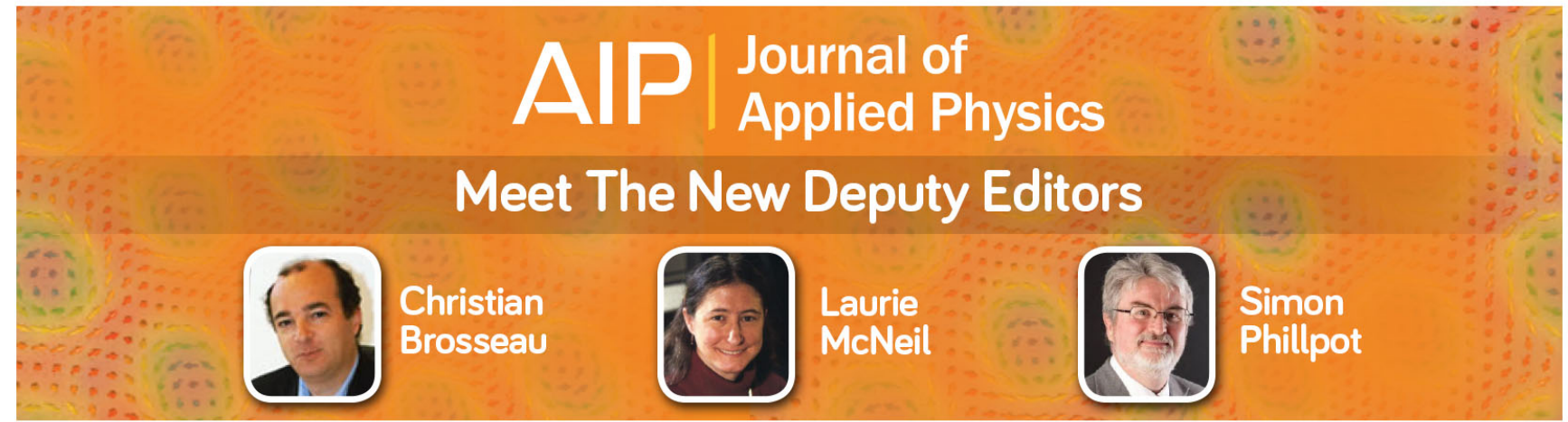




\title{
Magnetoelectric polymer nanocomposite for flexible electronics
}

\author{
M. Alnassar, ${ }^{\text {a) }}$ A. Alfadhel, Yu. P. Ivanov, and J. Kosel \\ Computer, Electrical and Mathematical Science and Engineering (CEMSE) Division, King Abdullah \\ University of Science and Technology, Thuwal 23955, Saudi Arabia
}

(Presented 5 November 2014; received 22 September 2014; accepted 30 October 2014; published online 6 March 2015)

\begin{abstract}
This paper reports the fabrication and characterization of a new type of magnetoelectric polymer nanocomposite that exhibits excellent ferromagnetism and ferroelectricity simultaneously at room temperature. The multiferroic nanocomposite consists of high aspect ratio ferromagnetic iron nanowires embedded inside a ferroelectric co-polymer poly(vinylindene fluoride-trifluoroethylene), $\mathrm{P}(\mathrm{VDF}-\mathrm{TrFE})$. The nanocomposite has been fabricated via a simple low temperature spin coating technique. Structural, ferromagnetic, ferroelectric, and magnetoelectric properties of the developed nanocomposite have been characterized. The nanocomposite films showed isotropic magnetic properties due to the random orientation of the iron nanowires inside the film. In addition, the embedded nanowires did not hinder the ferroelectric phase development of the nanocomposite. The developed nanocomposite showed a high magnetoelectric coupling response of $156 \mathrm{mV} / \mathrm{cmOe}$ measured at $3.1 \mathrm{kOe}$ DC bias field. This value is among the highest reported magnetoelectric coupling in two phase particulate polymer nanocomposites. (C) 2015 AIP Publishing LLC.

[http://dx.doi.org/10.1063/1.4913943]
\end{abstract}

\section{INTRODUCTION}

Multiferroic nanocomposites are of great interest for fundamental and technological aspects as they enable new functionalities and concepts for applications in sensors, actuators, data storage, or micro energy harvesting. The coupling between the constituent ferroic orders of these nanocomposite structures offers multifunctional properties and enhanced functionalities that are absent in each of the individual phases. Specifically, the coupling between ferroelectricity and ferromagnetism, the magnetoelectric effect (ME), brings about new potential applications in magnetoelectric sensors, energy harvesters, novel information storage, or smart ME devices. Composite ME materials show a spontaneous electrical polarization when subjected to a magnetic field, and an induced magnetization when subjected to an electrical field. Unlike ceramic-based ME composites, polymer-based multiferroic ME nanocomposites provide the advantages of being flexible, versatile, lightweight, low cost, able to conform to complicated shapes, and they typically involve a low-temperature fabrication process. These unique characteristics make polymer nanocomposites attractive for applications like flexible or wearable devices. Depending on the interface between the ferromagnetic and the polymer matrix, three main types of multiferroic polymer nanocomposites can be found in literature, particulate nanocomposite, laminate composites, and polymer as a binder composite. The first type received the least attention among researchers. ${ }^{1-4}$ Martins et al. reported incorporation of ferrite nanoparticles $(35-55 \mathrm{~nm})$ of $\mathrm{CoFe}_{2} \mathrm{O}_{4}$ or $\mathrm{Ni}_{0.5} \mathrm{Zn}_{0.5} \mathrm{Fe}_{2} \mathrm{O}_{4}$ into $\mathrm{P}(\mathrm{VDF}-\mathrm{TrFE})$ ferroelectric polymer matrix. In both cases, the nanocomposites exhibited improved ferroelectric, ferromagnetic, and ME properties, which were strongly

${ }^{\text {a)} E l e c t r o n i c ~ m a i l: ~ m o h a m m e d . a l n a s s a r @ k a u s t . e d u . s a ~}$ dependent on the ferrite filler concentrations. The highest ME response of Martins' reported nanocomposites was $41 \mathrm{mV} / \mathrm{cmOe}$ with $72 \mathrm{wt} . \%$ of $\mathrm{CoFe}_{2} \mathrm{O}_{4}$ at $2.5 \mathrm{kOe} \mathrm{DC}$ bias magnetic field. ${ }^{3,4}$ In this kind of multiferroic nanocomposites, the effective coupling properties are strongly dependent on the characteristics of the interface between the polymer and the ferromagnetic fillers. Surface interfacial area to volume ratio is a key factor and therefore high aspect ratio ferromagnetic nanostructures are preferred over spherical nanoparticles. This paper describes the development of a new type of multiferroic nanocomposite consisting of a ferroelectric copolymer poly(vinylidene fluoride-trifluoroethylene), $\mathrm{P}(\mathrm{VDF}-\mathrm{TrFE})$, and high aspect ratio ferromagnetic iron $(\mathrm{Fe})$ nanowires $(\mathrm{NWs})$ embedded inside the co-polymer. When subjected to a magnetic field, the strain transfer from the Fe NWs to the P(VDF-TrFE) matrix is maximized, owing to the large surface interfacial area of NWs with the polymer. This phenomena in the developed nanocomposite promises a strongly improved ME coupling when compared to low aspect ratio nanoparticles-based nanocomposites.

\section{EXPERIMENTAL PROCEDURES}

\section{A. Fabrication of ferromagnetic Fe NWs}

Anodic aluminum oxide (AAO) membranes offer selfassembled highly ordered hexagonal nanopores, which are ideal templates for homogenous nanoscale materials fabrication. Owing to their easy preparation, controllable pore size and spacing, as well as their low cost fabrication, nanowires, and nanotubes of polymers, metals, semiconductors, and composite materials have been fabricated successfully ${ }^{5-8}$ In this work, AAO membranes were prepared by a two-step anodization process using oxalic acid. ${ }^{9}$ First, highly pure Al discs with $500 \mu \mathrm{m}$ in thickness were electro polished for 
removal of the oxide layer and other contaminations. Then, the discs were exposed to a first anodization process in oxalic acid $(0.3 \mathrm{M})$ forming an alumina layer with randomly oriented pores. This random porous alumina layer is etched away using a chrome solution $(0.2 \mathrm{M}$ chromic acid and $0.4 \mathrm{M}$ phosphoric acid) leaving ordered dimples on the $\mathrm{Al}$ surface. Afterwards, a second anodization is applied and highly ordered hexagonal nanopores are obtained. Polycrystalline Fe nanowires are then grown inside these highly ordered nanopores by standard pulsed electrodeposition technique at room temperature. The electrolyte solution used contained $45 \mathrm{gl}^{-1}$ of iron sulfate, $30 \mathrm{gl}^{-1}$ of boric acid, and $1 \mathrm{gl}^{-1}$ of ascorbic acid mixed in DI water. Deposition pulses were negative with current density limit of $-60 \mathrm{~mA} / \mathrm{cm}^{2}$, and separated by positive polarization pulses and relatively long rest pulses to refresh the ion concentrations for homogenous deposition. ${ }^{10}$ The ferromagnetic Fe NWs were $6 \mu \mathrm{m}$ of length and $35 \mathrm{~nm}$ in diameter. Finally, Fe NWs were released from the membrane by dissolving the template membrane.

\section{B. Fabrication of multiferroic nanocomposite}

$\mathrm{P}(\mathrm{VDF}-\mathrm{TrFE})$, with $70 / 30 \%$ in molar ratio of VDF to TrFE, was dissolved in dimethylformamide (DMF) to form an 8 wt. \% solution. Fe NWs were dispersed in the same DMF solvent. The NWs were then added to the P(VDFTrFE) solution to get the polymer nanocomposite solution. The formed nanocomposite mixture had $1.5 \mathrm{vol} \% \mathrm{Fe}$ content. The solution was mixed thoroughly using a shaker followed by ultra-sonication for $10 \mathrm{~min}$ for dispersion and minimum NWs agglomeration. Thin film samples of the nanocomposite were obtained by spin coating at $1500 \mathrm{rpm}$ for $30 \mathrm{~s}$ on Si substrates with patterned Pt bottom electrodes obtained by standard lithography and lift off processes. After spinning, the nanocomposite film was baked at $80^{\circ}$ for 30 min for solvent evaporation. The ferroelectric $\beta$ phase of the polymer was facilitated by subsequent annealing in vacuum at $135^{\circ} \mathrm{C}$ for $2 \mathrm{~h}$. Then the films were allowed to gradually cool down to room temperature under vacuum. The thickness of the films was $950 \mathrm{~nm}$ measured using a stylus profiler (Dektak). For electrical and ME characterization, a $100 \mathrm{~nm}$ thick Au top electrode was thermally evaporated on top of the nanocomposite film. A one-mask photolithography process enabled Au patterning by a fast wet etching process using potassium iodide solution. The remaining photoresist on top of the $\mathrm{Au}$ patterns was removed with methanol.

\section{RESULTS AND DISCUSSION}

\section{A. Structural and magnetic characterization}

The crystallographic properties were investigated by Xray diffraction (XRD). The scan shows that the ferromagnetic Fe NWs used in the nanocomposite are polycrystalline with 200 preferred directions along their long axis (Fig. 1). The XRD result also shows the $\mathrm{P}(\mathrm{VDF}-\mathrm{TrFE})$ ferroelectric $\beta$ phase peak very clearly at $2 \theta$ of $19.8^{\circ}$, confirming the coexistence of the ferroelectric and ferromagnetic phases in the nanocomposite.

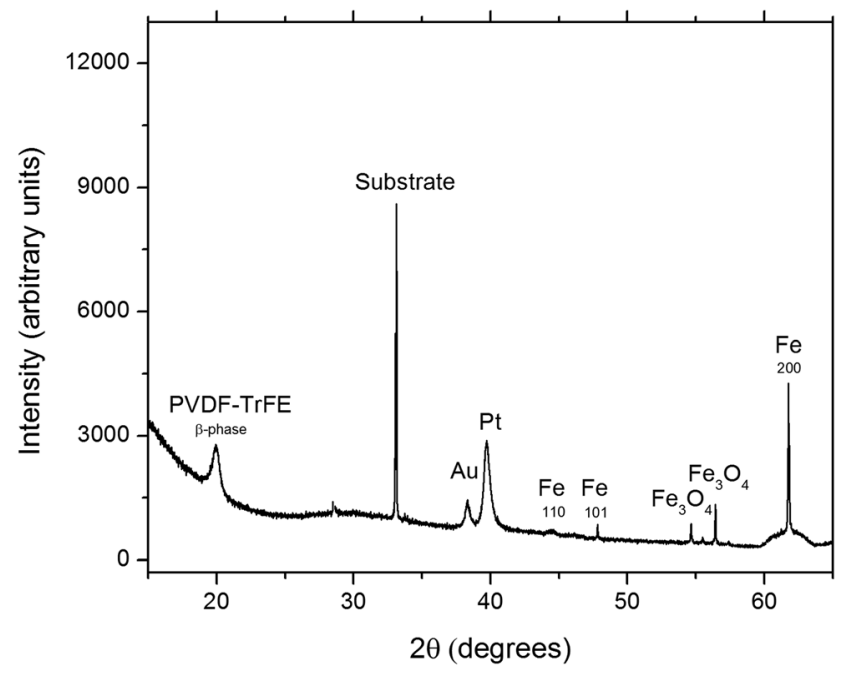

FIG. 1. XRD spectrum for the multiferroic nanocomposite film of P(VDFTrFE) with Fe NWs.

In order to investigate the magnetic properties, a vibrating sample magnetometer (VSM, Lakeshore) was used to measure the field dependent magnetization by applying a DC magnetic field between $-10 \mathrm{kOe}$ and $10 \mathrm{kOe}$ at room temperature. Fig. 2(a) shows the magnetization curve of Fe NWs inside the AAO template measured parallel, and perpendicular to the NW's axis. Clearly, the curve indicates an easy magnetization direction along the length of the wires, which is caused by the shape anisotropy of the high aspect ratio $\mathrm{Fe}$ wires. On the other side, the magnetization curve for the nanocomposite does not show field direction dependence (Fig. 2(b)), indicating that the Fe NWs are randomly oriented and well dispersed inside the ferroelectric polymer, and therefore the film is magnetically isotropic.

\section{B. Ferroelectric and magnetoelectric characterization}

To measure the ferroelectric polarization versus the electric field (P-E) of the developed nanocomposite, a precision multiferroic tester (Radiant Technologies) was used. The hysteresis loops were measured at room temperature with a maximum electric field of $125 \mathrm{MV} / \mathrm{m}$. By applying triangular waves oscillating at $10 \mathrm{~Hz}$ of frequency, welldefined ferroelectric loops were obtained for the fabricated nanocomposite sample (Fig. 3). When compared to pristine $\mathrm{P}(\mathrm{VDF}-\mathrm{TrFE})$ films, the measured ferroelectric loops indicate that the development of the ferroelectric $\beta$ phase is not hindered by the addition of the ferromagnetic Fe NWs.

Due to the dimensions of the NWs compared to the thickness of the film, all NWs are lying in-plane with random orientations as also confirmed by the magnetization curve (Fig. 2(b)). Therefore, the ME effect was characterized by measuring the longitudinal ME voltage coefficient $\alpha_{\mathrm{ME}}$ using the dynamic lock-in amplifier method. ${ }^{11}$ A pair of Helmholtz coils was used to generate an AC magnetic field with amplitude of $3 \mathrm{Oe}$ and frequency of $300 \mathrm{~Hz}$ that is superimposed to a DC bias field driven by an electromagnet. Both fields are applied in-plane of the nanocomposite film and the generated small signal voltage across the sample thickness is measured 

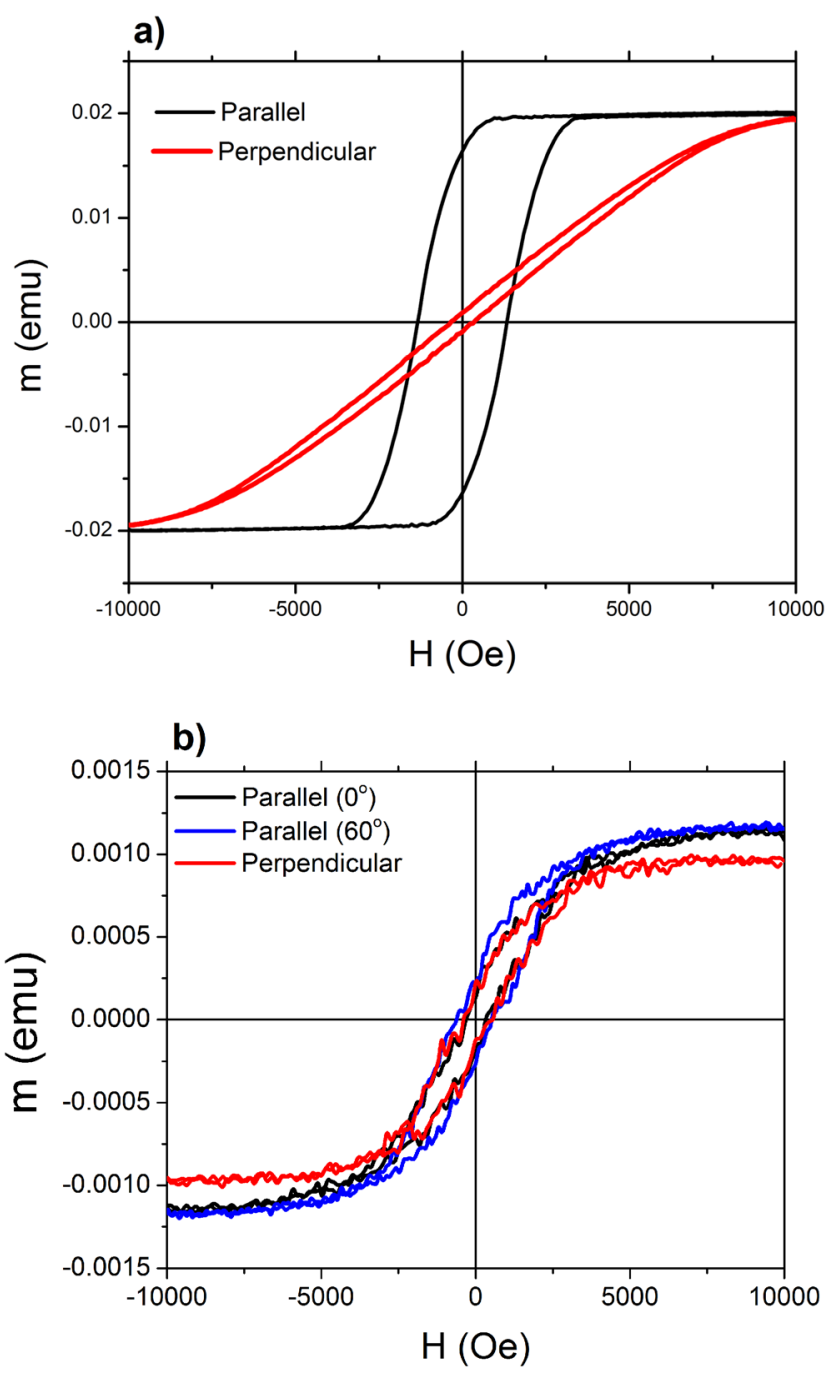

FIG. 2. Magnetic moment (m) as a function of applied field (H) of the (a) $\mathrm{Fe}$ NWs inside the AAO membrane and (b) the P(VDF-TrFE)/Fe NWs nanocomposite.

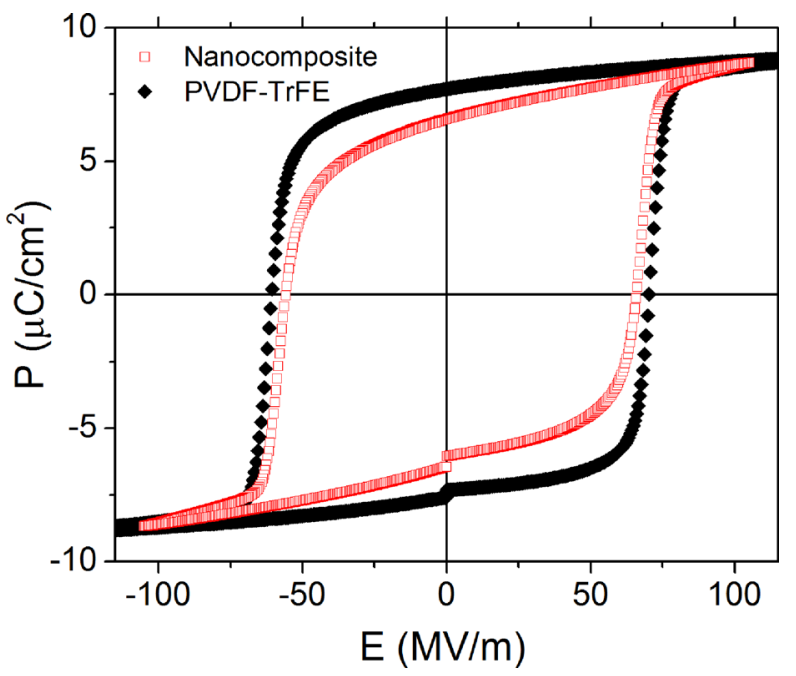

FIG. 3. Ferroelectric polarization (P) over electric field (E) measurements for the developed nanocomposite compared to a pristine $\mathrm{P}(\mathrm{VDF}-\mathrm{TrFE})$. using a digital Lock-in amplifier (Stanford Research SR850). The ME voltage coefficient $\alpha_{\mathrm{ME}}$ is calculated from the measured voltage using the equation $\alpha_{M E}(\mathrm{mV} / \mathrm{cmOe})$ $=\frac{V_{\text {out }}}{h_{0} \cdot t_{p}}$, where $V_{\text {out }}$ is the measured output voltage, $h_{o}$ is the amplitude of the AC magnetic field, and $t_{p}$ is the thickness of the nanocomposite film. ${ }^{6}$

Prior to ME characterization, the nanocomposite was electrically poled by applying a maximum electric field of $125 \mathrm{MV} / \mathrm{m}$. The ME voltage coefficient of the nanocomposite film, shown in Fig. 4, was measured by sweeping the DC field from 0 Oe to $3.5 \mathrm{kOe}$. A high ME response of $156 \mathrm{mV} / \mathrm{cmOe}$ was measured under $3.1 \mathrm{kOe}$ DC bias field. This value is considered among the highest reported for two phase particulate polymer nanocomposites, ${ }^{1,3}$ and it is attributed to the large interfacial area between the NWs and the polymer. The ME response curve also shows that the ME coupling is driven by a strain mediated effect and is mainly due to strain transfer caused by the non-linear magnetostriction effect of the Fe NWs. The drop in the ME curve afterwards is attributed to the saturation of the Fe NWs, where no further elongation or stress is present. The initial fluctuations in the ME voltage coefficient response is mainly due to the nature of the nanocomposite in which the Fe NWs are randomly aligned inside the ferroelectric polymer. Therefore, NWs, which are not in the same direction as the applied DC magnetic field cause torques and stresses depending on their distributions and angles with respect to the applied field. We expect this kind of initial fluctuations to be mitigated, when the NWs are aligned in a preferred direction while fabricating the nanocomposite.

\section{CONCLUSION}

A multiferroic polymer nanocomposite was successfully fabricated on a platinized Si substrate using P(VDF-TrFE) as the ferroelectric phase, and high aspect ratio Fe NWs as the ferromagnetic phase. Results showed that the addition of NWs to the P(VDF-TrFE) does not have a significant influence on the ferroelectric properties of the developed nanocomposite, and the nanocomposite exhibits both ferroelectric

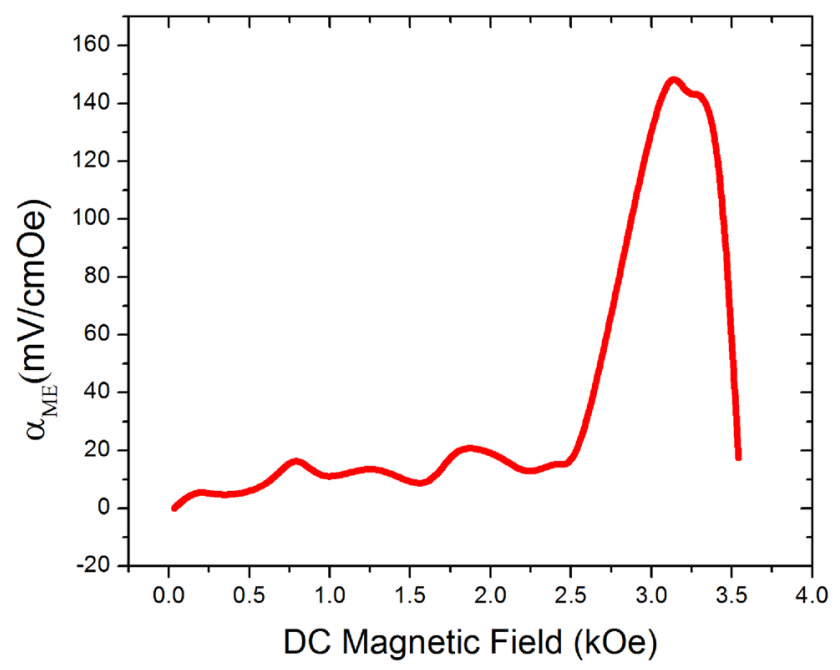

FIG. 4. ME voltage coefficient versus DC magnetic field bias of the nanocomposite. 
and ferromagnetic properties at room temperature. The use of high aspect ratio Fe NWs as ferromagnetic filler resulted in a ME coefficient up to $156 \mathrm{mV} / \mathrm{cmOe}$, which is higher than what has been achieved using ferrite nanoparticles. The enhancement is attributed to a higher interfacial area between the constituent phases. The developed nanocomposite is a promising candidate for flexible and smart $\mathrm{ME}$ applications.

\section{ACKNOWLEDGMENTS}

Research reported in this publication was supported by King Abdullah University of Science and Technology (KAUST). The authors also would like to acknowledge the financial support provided by SABIC.

${ }^{1}$ P. Martins and S. Lanceros-Mendez, "Polymer-based magnetoelectric materials," Adv. Funct. Mater. 23, 3371 (2013).

${ }^{2}$ J. Jin et al., "Multiferroic polymer composites with greatly enhanced magnetoelectric effect under low magnetic bias," Adv. Mater. 23, 3853 (2011).
${ }^{3} \mathrm{P}$. Martins et al., "Optimizing piezoelectric and magnetoelectric response on CoFe2O4/P(VDF-TrFE) nanocomposites," J. Phys. D: Appl. Phys. 44, 495303 (2011).

${ }^{4} \mathrm{P}$. Martins et al., "Linear anhysteretic direct magnetoelectric effect in $\mathrm{Ni0.5Zn0.5Fe2O4/poly(vinylidene} \mathrm{fluoride-trifluoroethylene)} \mathrm{0-3} \mathrm{nano-}$ composites," J. Phys. D: Appl. Phys. 44, 482001 (2011).

${ }^{5}$ D.-W. Shi et al., "Exchange-biased hybrid ferromagnetic-multiferroic core-shell nanostructures," Nanoscale 6, 7215-7220 (2014).

${ }^{6}$ A. Alfadhel et al., "Magnetic nanocomposite for biomimetic flow sensing," Lab Chip 14, 4362 (2014).

${ }^{7}$ A. B. Daly et al., "Synthesis and characterization of highly ordered cobalt-magnetite nanocable arrays," Small 2(11), 1299-1307 (2006).

${ }^{8} \mathrm{~V}$. Vega et al., "Tuning the magnetic anisotropy of $\mathrm{Co}-\mathrm{Ni}$ nanowires: Comparison between single nanowires and nanowire arrays in hard-anodic aluminum oxide membranes," Nanotechnology 23, 465709 (2012).

${ }^{9}$ H. Masuda and K. Fukuda, "Ordered metal nanohole arrays made by a two-step replication of honeycomb structures of anodic alumina," Science 268, 1466 (1995).

${ }^{10}$ K. Nielsch and B. Stadler, "Template-based synthesis and characterization of high-density ferromagnetic nanowire arrays," in Handbook of Magnetism and Advanced Magnetic Materials (Wiley \& Sons, 2007).

${ }^{11}$ G. V. Duong et al., "The lock in technique for studying ME effect," J. Magn. Magn. Mater. 316, 390 (2007). 